\title{
Anesthetic management of Congenital Adrenal Hyperplasia (CAH) in children - case report -
}

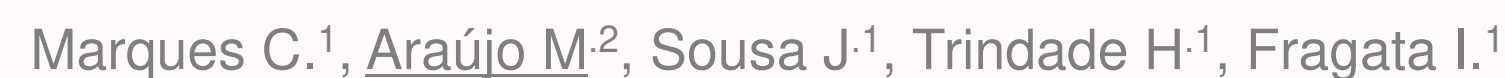

\author{
${ }^{1}$ Centro Hospitalar de Lisboa Central, Dept of Anaesthesiology, Lisboa, Portugal, \\ 2 Centro Hospitalar do Porto, Dept of Anaesthesiology \& Intensive Care, Porto, Portugal
}

\section{BACKGROUND}

- Autosomal recessive disorder associated with deficiencies in 21-,11-, or 17-hydroxylase (OHD)

- $>90 \%$ of $\mathrm{CAH}$ is caused by $21-\mathrm{OHD}$

- Clinical manifestations are mainly due to adrenocortical insufficiency, hyperandrogenism and adverse effects of glucocorticoids supplementation.

- Anaesthetic management surrounds the complications of ACTH suppression due to steroid therapy: hypertension, hyperglycemia, electrolyte abnormalities, adrenal and pituitary suppression and immune suppression.

\section{CASE REPORT}

- 20 month old female with masculinization due to CAH secondary to 21-OHD

- Medicated with hydrocortisone and fludrocortisone since birth.

\section{Surgery purposal: Elective reductive genitoplasty}

\section{Anesthetic technique: general-epidural anesthesia}

- Induction of the general anesthesia

- Epidural lumbar catheter placement - liquid column method (image 1)

- ropivacaine $0.2 \%$ was used according to anesthetic needs

- adrenal insufficiency protocol of our center was followed in the perioperative period:

$2 \mathrm{mg} / \mathrm{kg}$ of hydrocortisone in the anesthetic induction and every 4 hours during surgery In the first 24 post-operative hours, the dose is $20 \mathrm{mg}$ of intravenous hydrocortisone every 6 hours The habitual dose is resumed in the second post-operative day.

- Procedure lasted for 6 hours and 45 minutes (image 2)

- Vital parameters and glucose levels were stable

- Successfully extubation on operating room at the end of the surgery

- Transfer to pediatric intensive care unit

- Postoperative recovery was uneventful.

- Multimodal analgesia with epidural ropivacaine $0.1 \%$ infusion during 48 hours, with low pain scores.

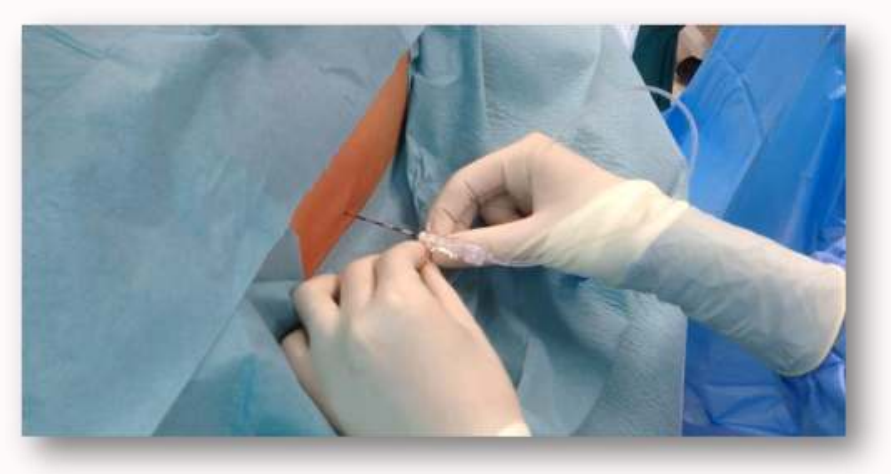

Image 1

Lumbar epidural cateter placement: liquid column method

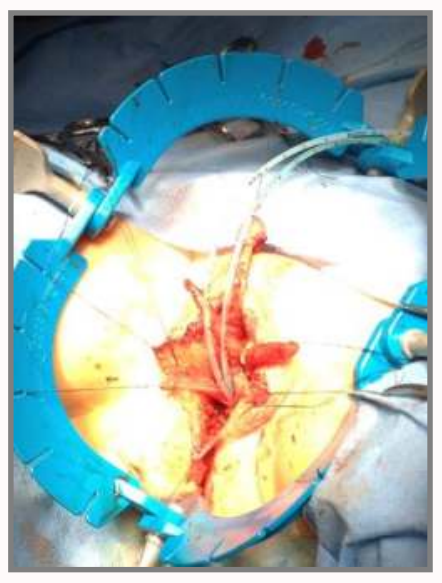

Image 2

Reductive genitoplasty in a female child with masculinization due to $21-O H D$

\section{DISCUSSION}

- Steroid supplementation is crucial in perioperative period

- Hemodynamic stability and good pain control were achieved during and after the procedure

- Epidural analgesia may contribute to this success.

\section{LEARNING POINTS}

Children with CAH can be managed successfully during anesthesia after understading the patophysiology of disease with proper history and perioperative steroid supplementation

- Hemodynamic and metabolic stability, as well a good pain control are major concerns in the anesthetic management of this patients. 